\title{
Erratum
}

\section{Deep Inelastic Scattering on a Photon Target in the Massive Quark Model}

P. Castorina, G. Nardulli, and G. Preparata

Z. Phys. C - Partıcles and Fields 8, 277 (1981)

The published paper contains two misprints. Equation (15) should be replaced by:

$$
\begin{aligned}
F_{2}^{\gamma}\left(x, Q^{2}\right)= & A_{\gamma} \frac{(1-x)^{3}}{3} F(2,1,4 ; 1-x) \\
& +B_{\gamma} \sqrt{x}(1-x)^{5 / 2} \frac{1}{5} F(1,2,7 / 2 ; 1-x) \\
& +C_{\gamma} x\left\{[-1+8 x(1-x)] \sqrt{1-\frac{4 m^{2} x}{Q^{2}(1-x)}}\right. \\
& \left.+\left[x^{2}+(1-x)^{2}\right] \ln \frac{Q^{2}(1-x)}{x m^{2}}\right\} .
\end{aligned}
$$

In Fig. 5 the curves $(a)$ and $(b)$ should be interchanged, as it is obvious from the $Q^{2}$-increase of $F_{2}\left(x, Q^{2}\right)$.

However such misprints do not alter in any way our conclusions. 